Transfer of coher ence and effect of I evel shi $\mathrm{ft}$ caused by two- quant um exci t at i on in a mul ti l evel NR syst em

\begin{tabular}{|l|l|}
\hline 著者 & Hat anaka H roshi, Hashi Tsuneo \\
\hline $\begin{array}{l}\text { j our nal or } \\
\text { publ i cat i on ti t l e }\end{array}$ & Physi cal Revi ew B \\
\hline vol une & 27 \\
\hline number & 7 \\
\hline page r ange & $4095-4109$ \\
\hline year & 1983 01- 01 \\
\hline URL & ht t p: //hdl . handl e. net /2297/7564 \\
\hline
\end{tabular}




\title{
Transfer of coherence and effect of level shift caused by two-quantum excitation in a multilevel NMR system
}

\author{
Hiroshi Hatanaka \\ Department of Physics, Faculty of Education, Kanazawa University, Kanazawa 920, Japan \\ Tsuneo Hashi \\ Department of Physics, Faculty of Science, Kyoto University, Kyoto 606, Japan \\ (Received 25 October-1982)
}

\begin{abstract}
The phenomenon of transfer of coherence and the effect of level shift caused by twoquantum (TQ) excitation were studied on ${ }^{27} \mathrm{Al}$ nuclei in $\mathrm{Al}_{2} \mathrm{O}_{3}$. Transfer of coherence between two single-quantum (SQ) transitions and that between SQ and three-quantum transitions were examined. It was observed that the coherences transfer periodically with frequency equal to one-half the TQ transient nutation frequency accompanied by phase shifts caused by the level shift. Experimental results are in good agreement with theoretical predictions. The effect of magnetic dipole interaction was also studied. A precise measurement was made on the shift of TQ resonance frequency and it was confirmed that no shift exists on the TQ resonance frequency contrary to the prediction based on the three-level model.
\end{abstract}

\section{INTRODUCTION}

Transfer of coherence is one of the fundamental phenomena in coherent spectroscopy. In a previous paper we reported the first investigation of this phenomenon in a multilevel NMR system and its application to the creation and detection of twoquantum (TQ) coherence. ${ }^{1}$ It has been shown that, when a single-quantum (SQ) transition is excited, the coherences in two nonresonant transitions which share a common level are transferred periodically with each other with frequency equal to one-half the transient nutation frequency of the SQ transition. This phenomenon can be pictorially represented by a vector precessing around an effective field in a special three-dimensional space. Coherence transfer rotary echoes ${ }^{2}$ are those associated with transfer of coherence.

The present paper is concerned with transfer of coherence caused by TQ excitation in a multilevel NMR system. The transfer of coherence caused by TQ excitation has been reported by Wolff and Mehring with a spin-1 system in connection with the spinor character of the spin system. ${ }^{3}$ We here present detailed studies of coherence transfer by TQ excitation on ${ }^{27} \mathrm{Al}$ nuclei $\left(I=\frac{5}{2}\right)$ in $\mathrm{Al}_{2} \mathrm{O}_{3}$ with emphasis on the effect of level shift which has not been examined so far. We examined two kinds of transfer phenomena caused by TQ excitation: One is coherence transfer between two SQ transitions, and the other that between SQ and three-quantum transitions. We observed that coherence transfer by TQ excitation occurs basically with frequency equal to one-half the TQ nutation frequency. In this respect the phenomenon is analogous to the coherence transfer by SQ excitation. However, the existence of level shift causes phase shifts in transferred coherences and makes the phenomenon unique and more complicated than that in the case of SQ excitation. The coherences observed with a phase-sensitive detection method exhibits complicated oscillation patterns reflecting the effect of level shift. By examining the phenomenon we could deduce the amount of the level shift caused by the TQ rf field. The origin of the level shift is similar to that of the ac Stark shift, ${ }^{4}$ well known in quantum optics, although magnetic dipole radiation is our concern here instead of the electric one. The effect of the level shift should exist in the case of the spin-1 system treated by Wolff and Mehring. ${ }^{3}$

It is well known that, when the TQ excitation is performed under the condition that the frequency offset to the intermediate state is much larger than Rabi frequencies, TQ coherent phenomena can be described by Bloch-type equations, ${ }^{5,6}$ and several TQ analogs of well-known SQ phenomena have been observed in NMR and quantum optics. ${ }^{7}$ Brewer and Hahn derived the equations applicable to the exact resonant TQ phenomena independent of the frequency offset to the intermediate state. ${ }^{8}$ However, 
Bloch-type equations and the equations derived by Brewer and Hahn for TQ coherent phenomena cannot explain the transfer phenomena caused by TQ excitation. We derived equations governing the coherence transfer by TQ excitation from our basic equation for TQ coherent phenomena in a multilevel NMR system. The effect of level shift is involved in the equations and the complicated oscillations of the observed coherences are explained as an effect of the level shift caused by TQ rf field. The effect of magnetic dipole interaction between $\mathrm{Al}$ nuclei is also taken into account to explain damping of the oscillation of the coherences. Experimental results are in good agreement with theoretical predictions.

We also examined the shift of TQ resonance frequency. In a three-level system usually used in quantum optics, the shift of TQ resonance frequency is expected and actually observed. ${ }^{9}$ In our case of the multilevel NMR system, no shift is expected from our basic equation, in contrast to the case of the three-level system. The difference comes from the fact that the effects of all levels in the multilevel NMR system are taken into account in our theory. We performed a precise measurement showing that no shift exists in TQ resonance frequency within experimental errors.

The theory of transfer of coherence caused by TQ excitation is given in Sec. II. The derivation of our basic equation for TQ coherent phenomena is given in the Appendix. In Sec. III the experimental procedure and apparatus are described. Section IV is divided into five subsections IV A-IVE. In the IV $A$ and IV B experimental results of the transfer phenomena are described and compared with the theory. In IV C the decay of the coherences due to magnetic dipole interaction is discussed and compared with experimental results. The effect of level shift on the transfer phenomena is treated in some detail in Sec. IV D. The last section, IV E, is concerned with the absence of the shift of TQ resonance frequency. The precise measurement proving this fact is described.

\section{THEORY OF COHERENCE TRANSFER CAUSED BY TQ EXCITATION}

We first present the basic equation for $T Q$ coherent phenomena which we derived previously. ${ }^{10}$ We consider quadrupolar nuclei with spin $I$ whose unperturbed Hamiltonian is given by

$$
\mathscr{H}_{0}=-\gamma H_{0} I_{z}+\omega_{q}\left[I_{z}^{2}-\frac{1}{3} I(I+1)\right],
$$

where $\gamma$ is the gyromagnetic ratio and $\hbar \omega_{q}$ is the electric quadrupole coupling energy. A static magnetic field $H_{0}$ is applied along the principal axis ( $z$ axis) of the electric field gradient (efg) tensor. This system has the energy levels with unequal spacings and no state mixing, and magnetic dipole transitions between levels with $\Delta m \geq 2$ are forbidden. The electric quadrupole coupling energy is assumed to be much smaller than the Zeeman energy $\hbar \gamma H_{0}$.

Suppose that an $\mathrm{rf}$ field $2 H_{1} \cos (\Omega t+\phi)$ is applied to the spin system along the $x$ axis in the laboratory frame. The frequency $\Omega$ is assumed to be nearly at TQ resonance and is far off resonance from singlequantum resonances. The time development of the spin system is described, in the interaction representation, by the equation of motion for the density matrix $\rho$ as

$$
\dot{\rho}(t)=i\left[\rho(t), \mathscr{H}_{1}(t)\right]
$$

with

$$
\begin{aligned}
\mathscr{H}_{1}(t)=- & 2 \gamma H_{1} \exp \left(i \mathscr{H}_{0} t\right) I_{x} \\
& \times \exp \left(-i \mathscr{H}_{0} t\right) \cos (\Omega t+\phi),
\end{aligned}
$$

where relaxation effects are neglected. We treat the case where $\gamma H_{1} \ll \omega_{q}$.

We use the following operators introduced previously, ${ }^{10}$ which are associated with eigenfunctions $|m\rangle,|n\rangle$ of $I_{z}$ as

$$
\begin{aligned}
& P_{m}=|m\rangle\langle m|, Q(m, n)=| m\rangle\langle n| \quad(m \neq n) \\
& Q_{x}(m, n)=Q(m, n)+Q(n, m), \\
& Q_{y}(m, n)=-i[Q(m, n)-Q(n, m)] \\
& Q_{z}(m, n)=P_{m}-P_{n}
\end{aligned}
$$

where $P_{m}$ is a projection operator on the state $m$. These operators are very useful in treating coherent phenomena in a multilevel system with unequal spacings. Some characteristics of these operators are shown in Eqs. (A3) in the Appendix. Essentially the same operators are used by several authors. ${ }^{11}$

Assuming the slowly varying amplitude approximation, we obtain the following basic equation for the TQ coherent phenomena:

$$
\begin{aligned}
\dot{\rho}^{\dagger}(t)= & i \beta\left[\rho^{\dagger}(t), Q_{x}(m+1, m-1) \cos (2 \phi)-Q_{y}(m+1, m-1) \sin (2 \phi)\right] \\
& +i \delta\left[\rho^{\dagger}(t), Q_{z}(m+1, m-1)\right]+i\left[\rho^{\dagger}(t), R\right],
\end{aligned}
$$

where we suppose that the transition between levels $m-1$ and $m+1$ is nearly at TQ resonance, i.e.,

$$
\Omega+\delta=\frac{1}{2}\left\{\left\langle m-1\left|\mathscr{H}_{0}\right| m-1\right\rangle-\left\langle m+1\left|\mathscr{H}_{0}\right| m+1\right\rangle\right\} \equiv \frac{1}{2} \Omega_{m-1, m+1},|\delta| \ll\left|\frac{1}{2} \Omega_{m-1, m+1}-\Omega\right|,
$$


and

$$
\begin{aligned}
& \rho^{\dagger}(t)=\exp \left[-i \delta Q_{z}(m+1, m-1) t\right] \rho(t) \exp \left[i \delta Q_{z}(m+1, m-1) t\right], \\
& \beta=\gamma^{2} H_{1}^{2}\left\langle m+1\left|I_{x}^{2}\right| m-1\right\rangle / \omega_{q} \\
& R=\gamma^{2} H_{1}^{2} \sum_{k}\left\langle k\left|I_{+}\right| k-1\right\rangle^{2} Q_{z}(k, k-1) / 4\left(\Omega-\Omega_{k-1, k}\right), \\
& \Omega_{k-1, k}=\left\langle k-1\left|\mathscr{H}_{0}\right| k-1\right\rangle-\left\langle k\left|\mathscr{H}_{0}\right| k\right\rangle
\end{aligned}
$$

The details of the derivation of Eq. (5) are given in the Appendix. The third term in Eq. (5) indicates the effect of level shift caused by the TQ-resonant $\mathrm{rf}$ field. Using the relations

$$
\begin{aligned}
& u_{m n}=\rho_{m n}^{\dagger}+\rho_{n m}^{\dagger}=\operatorname{Tr}\left[\rho^{\dagger} Q_{x}(n, m)\right], \\
& v_{m n}=-i\left(\rho_{n m}^{\dagger}-\rho_{m n}^{\dagger}\right)=-\operatorname{Tr}\left[\rho^{\dagger} Q_{y}(n, m)\right], \\
& w_{m n}=\rho_{n n}^{\dagger}-\rho_{m m}^{\dagger}=\operatorname{Tr}\left[\rho^{\dagger} Q_{z}(n, m)\right],
\end{aligned}
$$

and the commutation relations of $Q_{i}(m, n)$ $(i=x, y, z)$ shown in Eqs. (A3), we obtain equation of motion for the coherences $u_{m-1, m+1}, v_{m-1, m+1}$ and the population difference $w_{m-1, m+1}$ as follows:

$\dot{u}_{m-1, m+1}=2 \delta v_{m-1, m+1}-2 \beta w_{m-1, m+1} \sin 2 \phi$,

$\dot{v}_{m-1, m+1}=-2 \delta u_{m-1, m+1}+2 \beta w_{m-1, m+1} \cos 2 \phi$,

$\dot{w}_{m-1, m+1}=2 \beta u_{m-1, m+1} \sin 2 \phi-2 \beta v_{m-1, m+1} \cos 2 \phi$.

Equations (9) are rewritten in a form of a torque equation $\dot{\vec{r}}=-\overrightarrow{\mathrm{q}} \times \overrightarrow{\mathrm{r}}$ using vectors

$$
\overrightarrow{\mathrm{r}}=\left(u_{m-1, m+1}, v_{m-1, m+1}, w_{m-1, m+1}\right)
$$

and $\overrightarrow{\mathrm{q}}=[2 \beta \cos (2 \phi), 2 \beta \sin (2 \phi), 2 \delta]$. Then, in a reference frame rotating at frequency $2 \Omega$ around the $z$ axis, the vector $\vec{r}$ precesses at the frequency $2\left(\beta^{2}+\delta^{2}\right)^{1 / 2}$ around the effective field along the vector $\overrightarrow{\mathrm{q}}$, which tilts from the $z$ axis by an angle $\tan ^{-1}(\beta / \delta)$ and has an azimuthal angle $2 \phi$. The phase shift $\phi$ of the rf field brings about $2 \phi$ rotation of the effective field around the $z$ axis, and the off resonance $\delta$ appears as TQ off resonance of $2 \delta$. The vector model is the same as that obtained by Grischkowsky et al. ${ }^{6}$ The effect of the level shift does not appear in Eqs. (9). This is due to the fact that the operator $R$ commutes with $Q_{i}(m+1, m-1)$ $(i=x, y, z)$. The related experiment and discussions will be given in Sec. IV E.

Now we derive the equations representing the transfer of coherence caused by the TQ transition between levels $m-1$ and $m+1$. Hereafter, we assume that $\delta=\phi=0$. Using Eqs. (8) and the commutation relations for $Q$ operators shown in Eqs. (A3), the equations of motion for the coherences between levels $m \pm 1$ and $m+p$ are derived from Eq. (5) as

$$
\begin{aligned}
\dot{S}_{m \pm 1, m+p}= & i D(m, m+p) S_{m \pm 1, m+p} \\
& +i \beta S_{m \mp 1, m+p}
\end{aligned}
$$

with $S_{i j}=u_{i j}+i v_{i j}$, where $p$ is an integer $(\neq \pm 1)$. The coherences $S_{m+1, m+p}$ and $S_{m-1, m+p}$ are those corresponding to $|p-1|-$ and $|p+1|$-quantum transitions, respectively. The term $D(m, m+p)$ comes from the fact that the operators $Q_{i}(m \pm 1, m+p)(i=x, y)$ do not commute with the operator $R$ and is given by

$$
D(m, m+p)=\frac{2 \gamma^{2} H_{1}^{2}\left(p^{2}-1\right)\left(I^{2}-m^{2}+I+\frac{1}{4}\right)}{3 \omega_{q}\left(4 p^{2}-1\right)} .
$$

The solutions of Eq. (10) are

$$
\begin{aligned}
S_{m \pm 1, m+p}(t)= & u_{m \pm 1, m+p}(0) \\
& \times \exp [i D(m, m+p) t] \cos \beta t, \\
S_{m \mp 1, m+p}(t)= & u_{m \pm 1, m+p}(0) \\
& \times \exp \{i[D(m, m+p) t+\pi / 2]\} \\
& \times \sin \beta t,
\end{aligned}
$$

where it is assumed that only the coherence $u_{m-1, m+p}(0)$ or $u_{m+1, m+p}(0)$ exists before the application of the TQ pulse.

Equations (12) show that, when the TQ transition between levels $m-1$ and $m+1$ is excited (i.e., $\beta \neq 0$ ) the coherences $S_{m-1, m+p}$ and $S_{m+1, m+p}$ are no longer independent. If the effect of the term $D(m, m+p)$ is ignored, Eqs. (12) show the periodic transfer of coherence between $S_{m-1, m+p}(t)$ and $S_{m+1, m+p}(t)$ with frequency $\beta$ which is one-half the TQ transient nutation frequency. The fact that the transfer occurs at one-half the transient nutation frequency has been noted in the case of coherence transfer by SQ excitation. ${ }^{1}$ However, the existence of the term $D(m, m+p)$ in the present case makes the behavior more complicated than that in the case of SQ excitation. The value $D(m, m+p)$ causes a periodic phase variation of the coherences. This comes from the effect of the level shift, as will be 
described in Sec. IV D. The value of $D(m, m+p)$ is not necessarily small compared with the frequency $\beta$. If $\delta \neq 0$, Eq. (10) is modified to be

$$
\begin{aligned}
\dot{S}_{m \pm 1, m+p}= & i[D(m, m+p) \pm \delta] S_{m \pm 1, m+p} \\
& +i \beta S_{m \mp 1, m+p} .
\end{aligned}
$$

Equation (13) shows that the effect of $D(m, m+p)$ cannot be eliminated by introducing an appropriate amount of off-resonance $\delta$.

\section{EXPERIMENTAL PROCEDURE AND APPARATUS}

The experiment was performed on ${ }^{27} \mathrm{Al}$ nuclei in a single crystal of $\mathrm{Al}_{2} \mathrm{O}_{3}$. A static magnetic field $\mathrm{H}_{0}$ was applied along the principal axis ( $z$ axis) of the axially symmetric efg tensor at $\mathrm{Al}$ nuclei. The six levels of Al nuclei $\left(I=\frac{5}{2}\right)$ shown in Fig. 1 are eigenstates of $I_{z}$ and have unequal spacings. The intensity of the static magnetic field was adjusted so that the resonance frequency $\omega_{1}$ between levels $a$ and $b$ was $2 \pi \times 10.00 \mathrm{MHz}$. The resonance frequencies $\omega_{2}$ between levels $b$ and $c$, and $\omega_{4}$ between levels $c$ and $d$, were $2 \pi \times 9.64 \mathrm{MHz}$ and $2 \pi \times 9.29 \mathrm{MHz}$, respectively. We carried out two kinds of experiments of transfer of coherence caused by TQ excitation; $I$ is the transfer between SQ coherences and II is that between SQ and three-quantum coherences.

In experiment $I$, an if pulse with frequency $\frac{1}{2} \omega_{3}=\frac{1}{2}\left(\omega_{1}+\omega_{2}\right)$ was applied to excite TQ transition between levels $a$ and $c$ in the presence of the SQ coherence between levels $b$ and $c$, which was prepared by a $\pi / 2$ pulse with frequency $\omega_{2}$. The SQ coherence between levels $a$ and $b$ produced by the transfer of coherence was monitored by observing the amplitude of the free-induction decay (FID) sig-

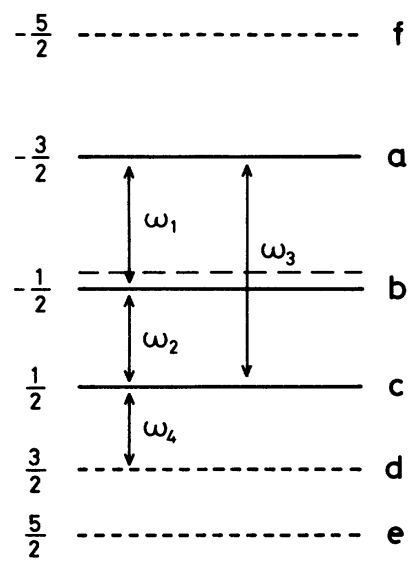

FIG. 1. Energy-level diagram of ${ }^{27} \mathrm{Al}$ nuclei in $\mathrm{Al}_{2} \mathrm{O}_{3}$. A static magnetic field $H_{0}$ is applied along the principal axis ( $z$ axis) of the efg tensor. nal at the frequency $\omega_{1}$ just after the TQ excitation pulse. The phase-sensitive detection (PSD) method was used to detect FID signals. The experimental apparatus is almost the same as described in a previous paper ${ }^{12}$ and not described here in detail. The rf sources used are two free-running oscillators with frequencies $\frac{1}{2} \omega_{1}$ and $\frac{1}{2} \omega_{2}$. The rf powers with frequencies $\omega_{1}$ and $\omega_{2}$ were obtained by frequency doubling, and that with frequency $\frac{1}{2} \omega_{3}$ by frequency mixing. After an appropriate amplification these powers were used to produce if fields. Thus all $\mathrm{rf}$ fields have fixed relative phases, and it enables us to use the PSD method for detecting FID signals.

In experiment II, the TQ transition between levels $b$ and $d$ was excited with an oscillator with frequency $\frac{1}{2}\left(\omega_{2}+\omega_{4}\right)$ in the presence of the SQ coherence between levels $a$ and $b$, which was prepared by a $\pi / 2$ pulse with frequency $\omega_{1}$. The effect of coherence transfer was detected by observing the amplitude of the FID signal at the frequency $\omega_{1}$ just after the TQ excitation pulse. The PSD method was used to detect the FID signal at the frequency $\omega_{1}$.

In these experiments we used a sample coil with low $Q$ for the excitation and detection at different frequencies. The amplitude $2 \mathrm{H}_{1}$ of the rf field for the TQ excitation was about 50 Oe. The linewidths of SQ transitions were about $2 \pi \times 8 \mathrm{kHz}$ and much smaller than the differences between resonance frequencies of adjacent SQ transitions. The frequency offset of the intermediate state was about $2 \pi \times 180$ $\mathrm{kHz}$ and was sufficiently larger than $\gamma H_{1}$.

The observations were made at $77 \mathrm{~K}$ to improve $S / N$ of the FID signals. We used a sample containing about $0.01 \% \mathrm{Cr}^{3+}$ ions to avoid the inconvenience due to very long $T_{1}$ in a pure sample. It was confirmed that the impurities did not affect the experimental results.

\section{EXPERIMENTAL RESULTS AND DISCUSSIONS}

\section{A. Transfer of coherence between SQ transitions $a-b$ and $b-c$}

The results of experiment $I$ are shown by open circles in Fig. 2. The amplitude of FID at $\omega_{1}$, which represents the degree of coherence between levels $a$ and $b$, is plotted as a function of the duration $\tau$ of the TQ excitation pulse. Figures 2(a) and 2(b) were taken at different $\mathrm{rf}$ phases of the reference signal in the PSD. In Fig. 2(a), the phase of the reference signal was chosen so that the FID signal at $\omega_{1}$, obtained by an application of a TQ $\pi / 2$ pulse with frequency $\frac{1}{2} \omega_{3}$ followed by a $\pi$ pulse (probe pulse) with frequency $\omega_{2}$ [denoted as $\frac{1}{2} \pi\left(\frac{1}{2} \omega_{3}\right) \pi\left(\omega_{2}\right)$ ], became maximum. In Fig. 2(b), the reference phase was 


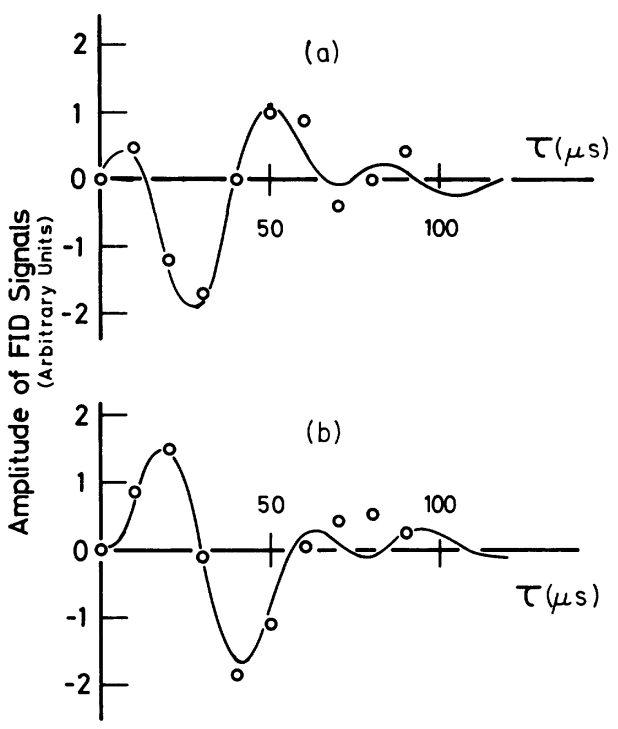

FIG. 2. Experimental and theoretical results indicating the transfer of coherence between SQ transitions $a-b$ and $b-c$ caused by TQ excitation of the transition $a-c$. Initially coherence $u_{b c}(0)$ is prepared and the transferred coherence between levels $a$ and $b$ is detected as a function of the duration $\tau$ of the TQ excitation pulse. Open circles in (a) and (b) show the observed coherences $v_{a b}$ and a mixture of $v_{a b}$ and $u_{a b}$, respectively. Oscillation patterns are not sinusoidal due to the effect of level shift caused by the TQ rf field. Solid curves are theoretical.

shifted by a certain amount.

Since the coherence is initially created only between levels $b$ and $c$, the experimental results in Fig. 2 show that the transfer of coherences between levels $a-b$ and $b-c$ occurs periodically by the TQ excitation. The oscillation patterns in Figs. 2(a) and 2(b) are complicated in contrast to the case of coherence transfer caused by SQ excitation, where sinusoidal oscillations are observed. The complication is due to the effect of the level shift described by the term $D(m, m+p)$ in Eqs. (12). This effect becomes detectable by using the PSD method. The term of $D(m, m+p)$ given by Eq. (11) can be rewritten as $35 \beta / 9 \sqrt{2}$ in the present case $\left(m=-\frac{1}{2}\right.$ and $\left.p=0\right)$ by using the expression of $\beta$ in Eqs. (7), and its value can be estimated from the observed TQ transient nutation frequency $2 \beta=2 \pi \times 13.6 \mathrm{kHz}$. The oscillatory behavior in Figs. 2(a) and 2(b) can be regarded as a superposition of the oscillations with frequencies $\beta$ and $D\left(-\frac{1}{2},-\frac{1}{2}\right)$.

In order to analyze the observed oscillation pattern, it is necessary to know the phase of the observed signals. In this paper, we use three reference frames $\left(x_{1}, y_{1}, z_{1}\right),\left(x_{2}, y_{2}, z_{2}\right)$, and $\left(x_{3}, y_{3}, z_{3}\right)$ rotating at the frequencies $\omega_{1}, \omega_{2}$, and $\omega_{3}$ with fixed relative phases. The coherences along the $x_{i}$ and $y_{i}$ $(i=1,2,3)$ axes are called $u$ and $v$ components, respectively. We assume that the rf field with frequency $\omega_{2}$ is applied along the $y_{2}$ axis. Then the coherence initially created between levels $b$ and $c$ should be $u_{b c}(0)$. The TQ pulse is assumed to be applied along the $x_{3}$ axis. Under this condition it can be shown, by using the commutation relations of $Q$ operators, that the coherence at the frequency $\omega_{1}$ produced by the operation $\frac{1}{2} \pi\left(\frac{1}{2} \omega_{3}\right) \pi\left(\omega_{2}\right)$ is $v_{a b}$. Therefore, the signal in Fig. 2(a) should be compared with the imaginary part of the following equation obtained from Eqs. (12),

$$
\begin{aligned}
S_{a b}(\tau)= & u_{b c}(0) \exp \left\{i\left[D\left(-\frac{1}{2},-\frac{1}{2}\right) \tau+\pi / 2\right]\right\} \\
& \times \sin (\beta \tau) .
\end{aligned}
$$

The coherence $u_{a b}(\tau)$ is also obtained from the real part of Eq. (14). The values of $\beta$ and $D\left(-\frac{1}{2},-\frac{1}{2}\right)$ estimated from the observed TQ transient nutation frequency are $2 \pi \times 6.8 \mathrm{kHz}$ and $2 \pi \times 18.7 \mathrm{kHz}$, respectively. The oscillation pattern in Fig. 2(a) is in good agreement with the expectation by Eq. (14) except for the damping. In the case of Fig. 2(b) a certain amount of $u_{a b}(\tau)$ is mixed. The detailed comparison is made in Sec. IV C after taking into account the effect of magnetic dipole interaction. The expression of $S_{b c}(\tau)$ is obtained from Eqs. (12) as

$$
S_{b c}(\tau)=u_{b c}(0) \exp \left\{-i\left[D\left(-\frac{1}{2},-\frac{1}{2}\right) \tau\right]\right\} \cos (\beta \tau) .
$$

It is to be noted that the signs in the exponents are different in Eqs. (14) and (15). This indicates that the effect of level shift is in opposite directions in $S_{a b}(\tau)$ and $S_{b c}(\tau)$.

Wolff and Mehring observed a similar phenomenon of transfer of coherence for a spin-1 system. ${ }^{3}$ The effect of level shift represented by $D(m, m+p)$ described above should exist in their case although they did not mention it. The value of $D(m, m+p)$ in their case is estimated to be

$$
D(0,0)=3 \gamma^{2} H_{1}^{2} / 2 \omega_{q}=3 \beta \text {. }
$$

\section{B. Transfer of coherence between SQ transition $a-b$ and three-quantum transition $a-d$}

The results of experiment II are shown by open circles in Fig. 3. The degree of coherence between levels $a$ and $b$ are plotted versus $\tau$. In Fig. 3(a), the phase of the reference signal in the PSD was adjusted so that the FID signal obtained by a $\pi / 2$ pulse at $\omega_{1}$ (along the $y_{2}$ axis) became maximum. The phase of the reference signal was shifted by $\pi / 2$ in Fig. 3(b). Therefore, the signals in Figs. 3(a) and 3(b) 


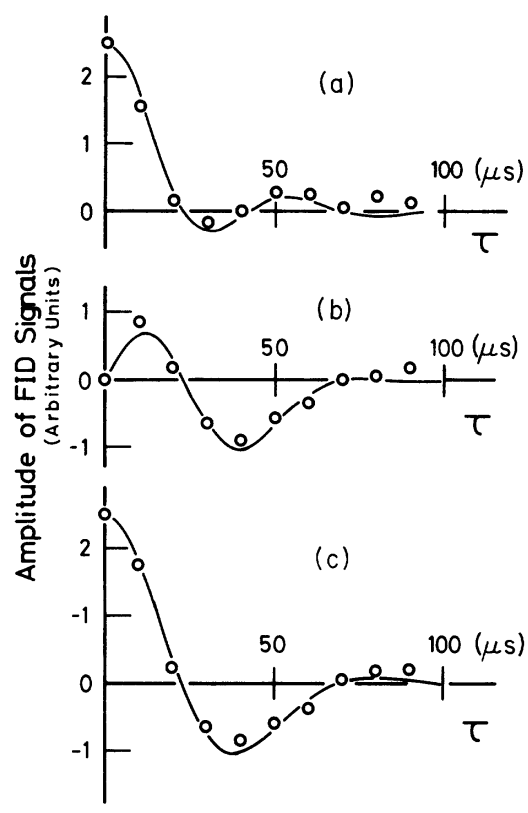

FIG. 3. Experimental and theoretical results indicating the transfer of coherence between the SQ transition $a-b$ and the three-quantum one $a-d$ caused by the TQ excitation of the transition $b-d$. The coherence $u_{a b}(0)$ is initially prepared and the changes of $u_{a b}$ and $v_{a b}$ vs $\tau$ (duration of the TQ excitation pulse) are plotted in (a) and (b), respectively. The effect of level shift caused by the TQ excitation is also seen in the oscillation patterns. (c) shows the vector sums of $u_{a b}$ and $v_{a b}$ where the effect of the level shift is eliminated. At $\tau \sim 23 \mu$ s and $\sim 68 \mu \mathrm{s}$, where the curve in (c) crosses the base line, the SQ coherence between levels $a$ and $b$ is completely transferred to the three-quantum one between levels $a$ and $d$. Solid lines are theoretical.

represent $u_{a b}(\tau)$ and $v_{a b}(\tau)$, respectively. The coherence initially created is $u_{a b}(0)$. Then the oscillations of the observed coherences in Fig. 3 show the periodic transfer of coherences. The transfer should occur between the SQ transition $a-b$ and the threequantum transition $a-d$, since the TQ transition between levels $b$ and $d$ is excited.

The appearance of the signal $v_{a b}(\tau)$ in Fig. 3(b) is due to the effect of level shift. The term $D\left(\frac{1}{2},-\frac{3}{2}\right)$ can be rewritten as $7 \beta / 9 \sqrt{2}$ by using Eqs. (11) and the expression of $\beta$ in Eqs. (7), which is smaller than $\beta$ in contrast to the case of experiment I. Therefore, the oscillation patterns are not as complicated as in Fig. 2. The positions of the first zeros in Figs. 3(a) and $3(b)$ are determined by the value of $\beta$. The appearance of the second zero in Fig. 3(a) at about 40 $\mu \mathrm{s}$ is due to the effect of a $D$ term. The expressions of $S_{a b}(\tau)$ and $S_{a d}(\tau)$ are derived from Eqs. (12),

$$
\begin{aligned}
S_{a b}(\tau)= & u_{a b}(0) \exp \left[-i D\left(\frac{1}{2},-\frac{3}{2}\right) \tau\right] \cos (\beta \tau), \\
S_{a d}(\tau)= & u_{a b}(0) \exp \left\{-i\left[D\left(\frac{1}{2},-\frac{3}{2}\right) \tau+\pi / 2\right]\right\} \\
& \times \sin (\beta \tau) .
\end{aligned}
$$

In contrast to Eqs. (14) and (15), the signs in the exponents in Eqs. (17) are the same, indicating that the effect of level shift is in the same direction.

Equations (12) suggest that the effect of $D$ term does not appear if one takes the vector sum of the signals in Figs. 3(a) and 3(b). Open circles in Fig. 3(c) represent such vector sums as a function of $\tau$. As is expected, the oscillation becomes sinusoidal except for the damping. From the oscillation frequency the value of $\beta$ is obtained to be $2 \pi \times 11 \mathrm{kHz}$. This value is also obtained from the TQ transient nutation frequency. Then the value of $D\left(\frac{1}{2},-\frac{3}{2}\right)$ becomes $2 \pi \times 6.0 \mathrm{kHz}$. At the zeros of Fig. 3(c) $(\sim 23,68 \mu \mathrm{s})$, the SQ coherence between levels $a$ and $b$ is completely transferred to the three-quantum transition $a-d$. The experimental curves in Fig. 3 are in good agreement with the theoretical ones obtained from Eqs. (17) except for the damping. The detailed comparison is made in Sec. IV C considering the effect of damping due to the effect of magnetic dipole interaction.

We tried to observe free decay of the threequantum coherence $a-d$ using an operation

$$
\frac{1}{2} \pi\left(\omega_{1}\right) \pi\left(\frac{1}{2} \omega_{2}+\frac{1}{2} \omega_{4}\right)-\tau-\pi\left(\frac{1}{2} \omega_{2}+\frac{1}{2} \omega_{4}\right),
$$

which is a two-step excitation consisting of SQ and TQ transitions followed by a probe pulse. However, the signal could only be detected near $\tau \sim 0$. This shows that the decay time of the three-quantum coherence is very short, which can also be predicted from the calculated values of second moments $M_{2}(a d)$ and $M_{2}(a b)$ obtained by using Eqs. (17) in our previous paper. ${ }^{12}$

C. Effect of magnetic dipole interaction on the coherence transfer

When the magnetic dipole interaction $\mathscr{H}_{d}$ is taken into account, the basic equation (5) is modified to be

$$
\begin{aligned}
\dot{\rho}^{\dagger}(t)= & i \beta\left[\rho^{\dagger}(t), \sum_{j} Q_{j x}(m+1, m-1) \cos (2 \phi)-\sum_{j} Q_{j y}(m+1, m-1) \sin (2 \phi)\right] \\
& +i \delta\left[\rho^{\dagger}(t), \sum_{j} Q_{j z}(m+1, m-1)\right]+i\left[\rho^{\dagger}(t), R\right]+i\left[\rho^{\dagger}(t), \mathscr{H}_{d}^{*}\right],
\end{aligned}
$$


with

$$
R=\gamma^{2} H_{1}^{2} \sum_{j, k}\left\langle k\left|I_{+}\right| k-1\right\rangle^{2} Q_{j z}(k, k-1) / 4\left(\Omega-\Omega_{k-1, k}\right),
$$

where $\mathscr{H}_{d}^{*}$ is the secular part of the dipole interaction. The derivation of Eq. (19) is given in the Appendix. The explicit form of $\mathscr{H}_{d}^{*}$ is given by ${ }^{12}$

$$
\mathscr{H}_{d}^{*}=\frac{1}{2} \sum_{i, j}\left[I_{i z} I_{j z}-\frac{1}{8} \sum_{m} \alpha_{m}^{2}\left[Q_{i x}(m, m-1) Q_{j x}(m, m-1)+Q_{i y}(m, m-1) Q_{j y}(m, m-1)\right]\right] D_{i j},
$$

with

$$
\begin{aligned}
& \alpha_{m}=\left\langle m\left|I_{+}\right| m-1\right\rangle, \\
& D_{i j}=\gamma^{2} \hbar\left(1-3 \cos ^{2} \theta_{i j}\right) / r_{i j}^{3},
\end{aligned}
$$

where $\overrightarrow{\mathrm{r}}_{i j}$ is a vector connecting nuclei $i$ and $j$, and $\theta_{i j}$ is an angle between $\overrightarrow{\mathrm{r}}_{i j}$ and the $z$ axis.

We consider the case where $\delta=0$. The amplitude $2 H_{1}$ of the TQ rf field is assumed to be so large that the dipole interaction can be treated as a perturbation compared with the Zeeman energy in the rotating reference frame. Only the time-independent part $\widetilde{\mathscr{H}}_{d}$ of

$$
\exp \left[i\left[\beta \sum_{j} Q_{j x}(m+1, m-1)+R\right] t\right] \mathscr{P}_{d}^{*} \text { (H.c.) }
$$

becomes effective on the decay of the coherence. The explicit form of $\tilde{\mathscr{H}}_{d}$ is given by

$$
\begin{aligned}
\tilde{\mathscr{H}}_{d}=\frac{1}{2} \sum_{i, j} & I_{i z} I_{j z}-Q_{i z}(m+1, m-1) I_{j z}-I_{i z} Q_{j z}(m+1, m-1) \\
& +\frac{3}{2} Q_{i z}(m+1, m-1) Q_{j z}(m+1, m-1)+\frac{1}{2} Q_{i y}(m+1, m-1) Q_{j y}(m+1, m-1) \\
& -\alpha_{m+2}^{2}\left[Q_{i x}(m+2, m+1) Q_{j x}(m+2, m+1)+Q_{i y}(m+2, m+1) Q_{j y}(m+2, m+1)\right] / 16 \\
& -\alpha_{m+2}^{2}\left[Q_{i x}(m+2, m-1) Q_{j x}(m+2, m-1)+Q_{i y}(m+2, m-1) Q_{j y}(m+2, m-1)\right] / 16 \\
& -\left(\alpha_{m+1}^{2}+\alpha_{m}^{2}\right)\left[Q_{i x}(m+1, m) Q_{j x}(m+1, m)+Q_{i y}(m+1, m) Q_{j y}(m+1, m)\right] / 16 \\
& -\left(\alpha_{m+1}^{2}+\alpha_{m}^{2}\right)\left[Q_{i x}(m, m-1) Q_{j x}(m, m-1)+Q_{i y}(m, m-1) Q_{j y}(m, m-1)\right] / 16 \\
& -\alpha_{m-1}^{2}\left[Q_{i x}(m-1, m-2) Q_{j x}(m-1, m-2)+Q_{i y}(m-1, m-2) Q_{j y}(m-1, m-2)\right] / 16 \\
& -\alpha_{m-1}^{2}\left[Q_{i x}(m+1, m-2) Q_{j x}(m+1, m-2)+Q_{i y}(m+1, m-2) Q_{j y}(m+1, m-2)\right] / 16 \\
& \left.-\frac{1}{8} \sum_{l} \alpha_{l}^{2}\left[Q_{i x}(l, l-1) Q_{j x}(l, l-1)+Q_{i y}(l, l-1) Q_{j y}(l, l-1)\right]\right) D_{i j}, \quad l \neq m \pm 1, m, m+2 .
\end{aligned}
$$

Since the Hamiltonian $\tilde{\mathscr{H}}_{d}$ commutes with $\sum_{j} Q_{j x}(m+1, m-1)$ and $R$, the solution of Eq. (19) is given by

$$
\rho(t)=\exp \left[-i\left[\beta \sum_{j} Q_{j x}(m+1, m-1)+R+\tilde{\mathscr{H}}_{d}\right] t\right] \rho(0)(\text { H.c. }) \text {. }
$$

We first consider the coherence transfer between SQ transitions $(m-1)-m$ and $m-(m+1)$ corresponding to experiment $\mathrm{I}$, where the coherence $u_{m, m+1}(0)$ is initially created between levels $m$ and $m+1$. In this case $\rho(0)$ is given by

$$
\rho(0)=A \sum_{j} Q_{j x}(m+1, m),
$$


where

$$
A=u_{m, m+1}(0) / \operatorname{Tr}\left[\sum_{j}\left[Q_{j x}(m+1, m)\right]^{2}\right] .
$$

Substituting Eq. (25) into

$$
u_{m-1, m}(t)=\operatorname{Tr}\left[\rho(t) \sum_{j} Q_{j x}(m, m-1)\right],
$$

we obtain

$$
\begin{aligned}
u_{m-1, m}(t)= & -A \Gamma_{1}(t) \sin [D(m, m) t] \sin (\beta t)+A \Gamma_{2}(t) \cos [D(m, m) t] \cos (\beta t) \\
& -A \Gamma_{3}(t) \cos [D(m, m) t] \sin (\beta t)+A \Gamma_{4}(t) \sin [D(m, m) t] \cos (\beta t),
\end{aligned}
$$

where

$$
\begin{aligned}
& \Gamma_{1}(t)=\operatorname{Tr}\left(\sum_{j} Q_{j x}(m, m-1) U_{1} \sum_{j} Q_{j x}(m, m-1) U_{1}^{-1}\right), \\
& \Gamma_{2}(t)=\operatorname{Tr}\left(\sum_{j} Q_{j x}(m, m-1) U_{1} \sum_{j} Q_{j x}(m+1, m) U_{1}^{-1}\right), \\
& \Gamma_{3}(t)=\operatorname{Tr}\left(\sum_{j} Q_{j x}(m, m-1) U_{1} \sum_{j} Q_{j y}(m, m-1) U_{1}^{-1}\right), \\
& \Gamma_{4}(t)=\operatorname{Tr}\left(\sum_{j} Q_{j x}(m, m-1) U_{1} \sum_{j} Q_{j y}(m+1, m) U_{1}^{-1}\right),
\end{aligned}
$$

with $U_{1}=\exp \left(-i \tilde{\mathscr{H}}_{d} t\right) . \Gamma_{1}(t)$ is an even function of $t$ since

$$
\begin{aligned}
\Gamma_{1}(t) & =\operatorname{Tr}\left(\sum_{j} Q_{j x}(m, m-1) U_{1} \sum_{j} Q_{j x}(m, m-1) U_{1}^{-1}\right) \\
& =\operatorname{Tr}\left(\sum_{j} Q_{j x}(m, m-1) U_{1}^{-1} \sum_{j} Q_{j x}(m, m-1) U_{1}\right)=\Gamma_{1}(-t) .
\end{aligned}
$$

Expanding $\Gamma_{1}(t)$ in power series of $t$ and assuming that it can be approximated by a Gaussian-type equation, we obtain

$$
\begin{aligned}
\Gamma_{1}(t)= & \operatorname{Tr}\left[\sum_{j}\left[Q_{j x}(m, m-1)\right]^{2}\right) \\
& \times \exp \left(-t^{2} / 2 T^{2}\right),
\end{aligned}
$$

where

$$
T^{-2}=\frac{-\operatorname{Tr}\left[\left[\tilde{H}_{d}, \sum_{j} Q_{j x}(m, m-1)\right]^{2}\right]}{\operatorname{Tr}\left[\sum_{j}\left[Q_{j x}(m, m-1)\right]^{2}\right]} .
$$

The other factors $\Gamma_{i}(t)(i=2,3,4)$ are shown to be zero. Therefore, Eq. (29) becomes

$$
\begin{aligned}
u_{m-1, m}(t)=- & u_{m, m+1}(0) \exp \left(-t^{2} / 2 T^{2}\right) \\
& \times \sin [D(m, m) t] \sin (\beta t) .
\end{aligned}
$$

In a similar way, we obtain

$$
\begin{aligned}
v_{m-1, m}(t)= & u_{m, m+1}(0) \exp \left(-t^{2} / 2 T^{2}\right) \\
& \times \cos [D(m, m) t] \sin (\beta t) .
\end{aligned}
$$

Thus

$$
\begin{aligned}
S_{m-1, m}(t)= & u_{m, m+1}(0) \exp \left(-t^{2} / 2 T^{2}\right) \\
& \times \exp \{i[D(m, m) t+\pi / 2]\} \\
& \times \sin (\beta t)
\end{aligned}
$$

For the coherence between levels $m$ and $m+1$, we obtain 


$$
\begin{aligned}
S_{m, m+1}(t)= & u_{m, m+1}(0) \exp \left(-t^{2} / 2 T^{2}\right) \\
& \times \exp [-i D(m, m) t] \cos (\beta t) .
\end{aligned}
$$

In the case of coherence transfer between the SQ transition $m-2-m-1$ and the three-quantum one $m-2-m+1$ as in experiment II, the following equations are derived in a manner similar to the previous case:

$$
\begin{aligned}
S_{m-2, m-1}(t)= & u_{m-2, m-1}(0) \exp \left(-t^{2} / 2 T^{2}\right) \\
& \times \exp [-i D(m, m-2) t] \cos (\beta t), \\
S_{m-2, m+1}(t)= & u_{m-2, m-1}(0) \exp \left(-t^{2} / 2 T^{2}\right) \\
& \times \exp \{-i[D(m, m-2) t+\pi / 2]\} \\
& \times \sin (\beta t),
\end{aligned}
$$

where $T$ are obtained from Eq. (33) by replacing $m$ by $m-1$.

We also take into account of the effect of the dead time $\tau_{d}$ of the receiver because the damping during $\tau_{d}$ is not negligible. Since the time development during $\tau_{d}$ is governed by $U_{2}=\exp \left(-i \mathscr{H}_{d}^{*} \tau_{d}\right)$, we have

$$
\rho\left(t+\tau_{d}\right)=U_{2} \rho(t) U_{2}^{-1}
$$

where $\rho(t)$ is given by Eq. (25). Using Eq. (39) instead of Eq. (25), we obtain

$$
\begin{aligned}
S_{a b}\left(\tau+\tau_{d}\right)= & u_{b c}(0) \exp \left[-\left(\tau+E \tau_{d}\right)^{2} / 2 T^{2}\right] \sin (\beta \tau) \\
& \times \exp \left\{i\left[D\left(-\frac{1}{2},-\frac{1}{2}\right) \tau+\pi / 2\right]\right\},
\end{aligned}
$$

instead of Eq. (36), with

$$
E=\frac{\operatorname{Tr}\left(\left[\mathscr{H}_{d}^{*}, \sum_{j} Q_{j x}(b, a)\right]\left[\tilde{\mathscr{H}}_{d}, \sum_{j} Q_{j x}(b, a)\right]\right]}{\operatorname{Tr}\left(\left[\tilde{\mathscr{H}}_{d}, \sum_{j} Q_{j x}(b, a)\right]^{2}\right]},
$$$$
T^{-2}=\frac{-\operatorname{Tr}\left[\left[\tilde{\mathscr{H}}_{d}, \sum_{j} Q_{j x}(b, a)\right]^{2}\right]}{\operatorname{Tr}\left(\sum_{j}\left[Q_{j x}(b, a)\right]^{2}\right]},
$$

where we replace the notations $m-1, m$, and $m+1$ by $a, b$, and $c$, respectively, in accordance with the experimental condition. In the case of $\tau=0, E$ becomes unity and $T$ gives the decay time of the FID associated with the coherence between levels $a$ and $b^{12}$

The solid curve in Fig. 2(a) represents $v_{a b}$ obtained from Eq. (40), where the value of $\tau_{d}$ is taken to be $30 \mu \mathrm{s}$, and the amplitude is adjusted so that the best fit to the experimental one is obtained. The solid line in Fig. 2(b) is drawn by introducing a phase shift of $7 \pi / 18$ with respect to the curve in Fig. 2(a). These theoretical curves are in good agreement with the experimental ones for $\tau \lesssim 60 \mu \mathrm{s}$. The disagreement for $\tau>60 \mu \mathrm{s}$ is considered to be due to the undesirable excitation of the transition $a-b$ by the TQ rf pulse.

The theoretical expressions which should be compared with the experimental results in Fig. 3 is obtained in a similar way as

$$
\begin{aligned}
S_{a b}\left(\tau+\tau_{d}\right)= & u_{a b}(0) \exp \left[-\left(\tau+E \tau_{d}\right)^{2} / 2 T^{2}\right] \\
& \times \cos (\beta \tau) \exp \left[-i D\left(\frac{1}{2},-\frac{3}{2}\right) \tau\right],
\end{aligned}
$$

where $E$ and $T$ are obtained from Eqs. (41) by using the time-independent part of

$$
\exp \left[i\left[\beta \sum_{j} Q_{j x}(d, b)+R\right] t\right] \mathscr{C}_{d}^{*} \exp \left[-i\left[\beta \sum_{j} Q_{j x}(d, b)+R\right] t\right]
$$

as $\tilde{\mathscr{H}}_{d}$. The solid curves in Figs. 3(a) and 3(b) represent $u_{a b}(\tau)$ and $v_{a b}(\tau)$ obtained from Eq. (42), respectively, where the amplitude $u_{a b}(0)$ is adjusted so that it coincides with the experimental value.

The solid curve in Fig. 3(c) represents

$$
\begin{aligned}
\left(u_{a b}^{2}+v_{a b}^{2}\right)^{1 / 2}= & u_{a b}(0) \exp \left[-\left(\tau+E \tau_{\alpha}\right)^{2} / 2 T^{2}\right] \\
& \times \cos (\beta \tau),
\end{aligned}
$$

which shows the sinusoidal oscillation with damp- ing. The agreement between theoretical and experimental curves is quite satisfactory.

\section{Effect of the level shift on transfer of coherence due to TQ excitation}

In this section we discuss the effect of the level shift in the coherence transfer caused by TQ excitation. The operator $R$ in the basic equation (5) represents the level shift caused by the TQ rf field. 
In the case of TQ exact resonance $(\delta=0)$, the effective Hamiltonian in the interaction representation where the effect of $\mathscr{H}_{0}$ is eliminated, is derived from Eq. (5) as

$$
\mathscr{H}=\beta Q_{x}(m+1, m-1)+R,
$$

where we assume that $\phi=0$ for simplicity. Since the TQ $\mathrm{rf}$ field is assumed to be far off resonance with SQ transitions, the level shift $\Delta_{n}$ (in angular frequency units) of the state with magnetic quantum number $n$ is given by

$$
\begin{aligned}
\Delta_{n}=\operatorname{Tr}\left(\mathscr{H} P_{n}\right)= & \operatorname{Tr}\left(R P_{n}\right) \\
= & \epsilon_{n}^{2} /\left(\Omega-\Omega_{n-1, n}\right) \\
& -\epsilon_{n+1}^{2} /\left(\Omega-\Omega_{n, n+1}\right),
\end{aligned}
$$

where

$$
\epsilon_{n}=\frac{1}{2} \gamma H_{1} \alpha_{n} .
$$

The first and second terms in the right-hand side of Eq. (46) show the shifts of level $n$ caused by the virtual transitions $n-1-n$ and $n-n+1$, respectively. The shift occurs in the direction such that the amount of off resonance in each transition is increased.

The shifts $\Delta_{m \pm 1}$ and $\Delta_{m}$ of the levels $m \pm 1$ and $m$ caused by the TQ rf field exactly at resonance with TQ transition $m+1 \leftrightarrow m-1$ are obtained from Eq. (46) as

$$
\begin{aligned}
& \Delta_{m \pm 1}=\operatorname{Tr}\left(R P_{m \pm 1}\right)=\gamma^{2} H_{1}^{2}\left(I^{2}-m^{2}+I+1\right) / 6 \omega_{q}, \\
& \Delta_{m}=\operatorname{Tr}\left(R P_{m}\right)=-\gamma^{2} H_{1}^{2}\left(I^{2}-m^{2}+I\right) / 2 \omega_{q} .
\end{aligned}
$$

Then the transition frequency between levels $m-1$ and $m$ becomes higher by an amount

$$
\begin{aligned}
\zeta_{m-1, m} & =\Delta_{m-1}-\Delta_{m} \\
& =2 \gamma^{2} H_{1}^{2}\left(I^{2}-m^{2}+I+\frac{1}{4}\right) / 3 \omega_{q},
\end{aligned}
$$

whereas the transition frequency between levels $m$ and $m+1$ becomes lower by the same amount $\left(\zeta_{m, m+1}=-\zeta_{m-1, m}\right)$.

The transfer of coherence occurs in the presence of the level shift and therefore the phases of the relevant coherences are shifted after the transfer, i.e., the effect of level shift appears as the phase shifts of the transferred coherences. The phase of the observed coherence between levels $m-1$ and $m$ advances, and that between levels $m+1$ and $m$ delays by the same amount. The values of $\zeta_{m-1, m}$ and $\zeta_{m, m+1}$ are equal to those of $D(m, m)$ and $-D(m, m)$ estimated from Eq. (11), respectively. The effect of level shift described above is involved in Eqs. (14) and (15), where the term $D(m, m)$ has different signs.

The amount of the level shifts $\Delta_{b}, \Delta_{a}$, and $\Delta_{c}$ can be obtained from the observed value of $D\left(-\frac{1}{2},-\frac{1}{2}\right)$ or $\beta$ with the aid of Eq. (48). The result is $\Delta_{b}=-2 \pi \times 13.6 \mathrm{kHz}$ and $\Delta_{a}=\Delta_{c}=2 \pi \times 5.1 \mathrm{kHz}$ for $2 H_{1}=43$ Oe.

In the case of coherence transfer between SQ and three-quantum transitions, the level shifts by the TQ rf field produce the phase shifts of coherences in the same directions as is seen from Eqs. (17), where the signs in the exponents are the same. This fact can be understood intuitively because the level $d$ is outside the levels relevant to the TQ transition. The amount of the level shifts $\Delta_{a}, \Delta_{b}\left(=\Delta_{d}\right)$, and $\Delta_{c}$ are obtained as $2 \pi \times 2.2 \mathrm{kHz}, 2 \pi \times 8.2 \mathrm{kHz}$, and $-2 \pi \times 22.0 \mathrm{kHz}$, respectively, for $2 H_{1}=55$ Oe from the observed value of $D\left(\frac{1}{2},-\frac{3}{2}\right)$ or $\beta$ with the aid of Eqs. (48) and

$$
\Delta_{m-2}=\gamma^{2} H_{1}^{2}\left(I^{2}-m^{2}+I+4\right) / 30 \omega_{q},
$$

where $m=\frac{1}{2}$.

\section{E. Shift of TQ resonance frequency}

In previous sections we have shown that level shifts are produced by the TQ rf field. However, Eqs. (48) show an interesting fact-that the level shifts $\Delta_{m+1}$ and $\Delta_{m-1}$ are equal, and therefore no frequency shift is expected for the TQ resonance frequency. In our previous experiments on TQ coherent phenomena, such as the TQ transient nutation, etc., ${ }^{13}$ no evidence was observed on the shift of TQ resonance frequency, consistent with this prediction.

However, in a pure three-level system, the shift of the TQ resonance frequency is expected. If we take a three-level system consisting of levels $m-1, m$, and $m+1$, it is expected that the TQ resonance frequency is shifted by an amount

$$
\zeta=\gamma^{2} H_{1}^{2}\left(\alpha_{m+1}^{2}-\alpha_{m}^{2}\right) / 4 \omega_{q},
$$

which is generally not zero (it becomes zero in the special case of $m=0$ ). This formula can also be derived from the exact pulse solutions of Brewer and $\mathrm{Hahn}^{8}$ for the TQ coherent phenomena when the large frequency offset of the intermediate state is assumed.

The calculated value of $\zeta$ is usually very small in the system consisting of three levels in a multilevel NMR system whose Hamiltonian is given by Eq. (1). Therefore, the three-level model is a good approximation in the present experiment. However, as will be described below, a high-precision measurement shows that the shift (51) predicted by the three-level 
model does not agree with experimental results.

The experiment was performed as follows. First, we fixed the strength of the static magnetic field $H_{0}$ and measured the resonance frequency $v_{1}$ between levels $f$ and $a$ very precisely, using a method which we reported previously. ${ }^{14}$

The method is illustrated in the lower part in Fig. 4. We apply a long pulse whose amplitude is initially small (about $0.01 \mathrm{Oe}$ ) and adiabatically increased to about 20 Oe. If the rf field is slightly offresonance, the FID signal should be observed just after the pulse. Plotting the amplitudes of the FID signal as a function of the frequency of the rf field, we obtain a curve like in Fig. 4 . The point where the curve crosses the base line gives the value of the resonance frequency. This method gives the exact resonance frequency of the spins whenever the first moment of the absorption line is zero, i.e., even when the resonance is asymmetrically broadened.

Next, we measured the resonance frequency between levels $a$ and $b v_{2}$ by the same method. The experimental results for the measurement of $v_{2}$ is shown in Fig. 4. The resonance frequencies obtained are $v_{1}=9999.8 \pm 0.2 \quad \mathrm{kHz}$ and $v_{2}=9644.0 \pm 0.2 \mathrm{kHz}$, respectively. From these values the (unshifted) resonance frequency between levels $f$ and $b$ is given by $v_{1}+v_{2}=19643.8 \pm 0.4$ kHz.

Finally, we measured the TQ resonance frequency
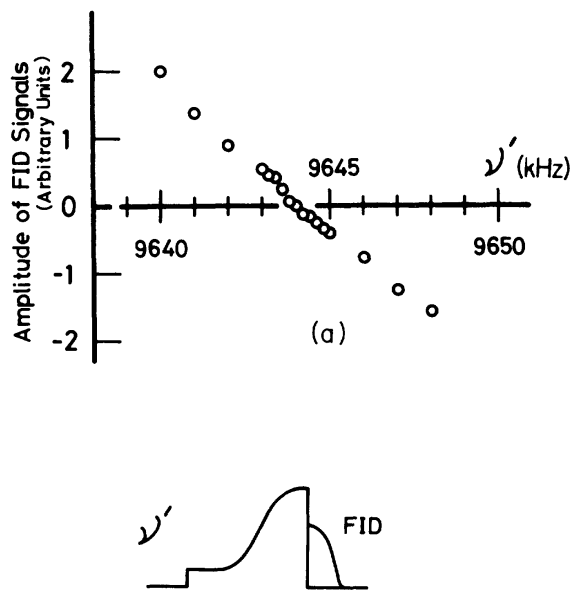

(b)

FIG. 4. A method for precise determination of the SQ resonance frequency (b) and the experimental result obtained by this method (a). The amplitude of the rf field is adiabatically increased from about $0.01 \mathrm{Oe}$ to about $20 \mathrm{Oe}$ and turned off suddenly. Open circles show the initial amplitudes of FID signals just after the rf pulse for various values of $\mathrm{rf}$ frequency $v^{\prime}$. The point where the curve crosses the base line gives the exact resonance frequency $v_{2}$. with an operation as shown in Fig. 5(b). We apply a long TQ excitation pulse with frequency $v$ [ $\left.\sim \frac{1}{2}\left(v_{1}+v_{2}\right)\right]$ followed by a $\pi$ pulse with frequency $v_{2}$. The duration of the TQ excitation pulse is so long that a quasi-steady state of the spin system is achieved in the rotating reference frame. It can be shown that when the frequency $v$ shifts by $\Delta / 2 \pi$ from the TQ resonant frequency, the TQ effective field is tilted from the $z$ axis by an angle

$$
\theta=\tan ^{-1}(\beta / \Delta)
$$

in the reference frame rotating at the frequency $2 v$. In this case, a TQ coherence proportional to $\cos \theta \sin \theta$ is expected to be created between levels $f$ and $b$ as a dispersion component. The $\pi$ pulse (probe pulse) with frequency $v_{2}$ detects this dispersion component of TQ coherence as a FID signal between levels $f$ and $a$. Plotting the initial amplitudes of the FID signals as a function of $v$, we obtained the curve shown in Fig. 5. The TQ resonance frequency is obtained to be $v_{0}=9822.2 \pm 0.2 \mathrm{kHz}$ from the crossing point to the base line. In this measurement undesirable signals arising from the direct excitation by the long TQ pulse are eliminated since the probe pulse selectively detects the TQ coherence.

The shift of the TQ resonance frequency between levels $f$ and $b$ is then $v_{1}+v_{2}-2 v_{0}=-0.6 \pm 0.8 \mathrm{kHz}$,
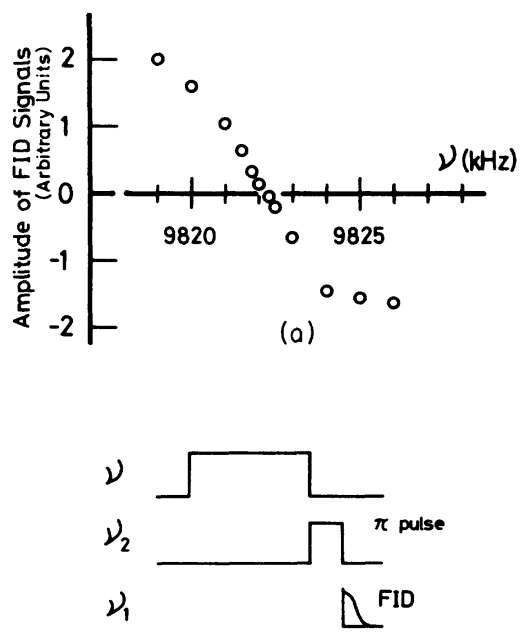

(b)

FIG. 5. A method for precise determination of the TQ resonance frequency under the irradiation of the TQ $\mathrm{rf}$ field and the result obtained by this method. Open circles indicate the amplitudes of FID signals after the $\pi$ (probe) pulse for various frequencies of the TQ rf field. The amplitudes are proportional to the magnitudes of the dispersion components of the TQ coherence. The crossing point of the curve and the base line gives the TQ resonance frequency. 
which should be compared with the calculated value of $\zeta / 2 \pi$. The three-level model [Eq. (51)] gives the value

$$
\zeta / 2 \pi=3.0 \pm 0.1
$$

in units of $\mathrm{kHz}$, for the TQ rf field used $\left(2 H_{1}=48\right.$ Oe), where $I=\frac{5}{2}$ and $m=-\frac{3}{2}$. The experimental value of $\zeta / 2 \pi$ is clearly different from the theoretical one and can be regarded as zero within experimental errors. Therefore, we conclude that the three-level model gives incorrect results for the calculation of the shift of TQ resonance frequency. ${ }^{15}$ Our Eqs. (48) give the correct value of the shift; in other words, one should take into account not only the effect of intermediate state but also those of other nonresonant levels in a multilevel system in order to calculate level shift due to the TQ rf field.

\section{APPENDIX}

The Hamiltonian $\mathscr{H}_{1}(t)$ given by Eq. (3) is a fastoscillating operator since the frequency $\Omega$ is far off resonance from SQ resonances. Assuming the slowly varying amplitude approximation, we expand the right-hand side of Eq. (3) by using integration by parts. The first-order term is fast oscillating, and the third- and higher-order terms are small compared to the second-order one. Therefore, we obtain

$$
\dot{\rho}(t)=-\left[\left[\rho(t), \int_{0}^{t} \mathscr{H}_{1}\left(t^{\prime}\right) d t^{\prime}\right], \mathscr{H}_{1}(t)\right] .
$$

We develop the right-hand side of Eq. (A1) by applying projection operator $\sum_{m} P_{m}$ on both sides of the Hamiltonian $\mathscr{H}_{1}(t)$ and rewrite it by using the relations

$$
\begin{aligned}
& I_{+}=\sum_{m} P_{m} I_{+} \sum_{m} P_{m}=\sum_{m} \alpha_{m} Q(m, m-1), \\
& I_{-}=\sum_{m} P_{m} I_{-}-\sum_{m} P_{m}=\sum_{m} \alpha_{m} Q(m-1, m), \\
& I_{z}=\sum_{m} P_{m} I_{z} \sum_{m} P_{m}=\frac{1}{2} \sum_{m>n} \sum_{z}(m, n), \\
& I_{x, y}=\frac{1}{2} \sum_{m} \alpha_{m} Q_{x, y}(m, m-1),
\end{aligned}
$$

with $\alpha_{m}=\left\langle m\left|I_{+}\right| m-1\right\rangle$ and following characteristics of the operators $P_{m}, Q(m, n)$, and $Q_{i}(m, n)$ $(i=x, y, z)$,

$$
\begin{aligned}
& P_{m} P_{n}=\left\{\begin{array}{l}
P_{m} \text { if } m=n \\
0 \text { if } m \neq n,
\end{array}\right. \\
& Q(k, j) Q(n, m)=\left\{\begin{array}{l}
P_{m} \text { if } j=n \text { and } k=m \\
Q(k, m) \text { if } j=n \text { and } k \neq m \\
0 \text { if } j \neq n,
\end{array}\right. \\
& {\left[Q_{p}(m, n), Q_{q}(m, n)\right]=2 i Q_{r}(m, n), p, q, r=z, y, z \text { (cyclic permutation), }} \\
& {\left[Q_{p}(m, n), Q_{p}(m, k)\right]=\left\{\begin{array}{l}
i Q_{y}(n, k) \text { if } p=x, y \\
0 \text { if } p=z,
\end{array}\right.} \\
& {\left[Q_{x}(m, n), Q_{y}(m, k)\right]=-i Q_{x}(n, k), \quad\left[Q_{y}(m, n), Q_{z}(m, k)\right]=i Q_{x}(m, n),} \\
& {\left[Q_{z}(m, n), Q_{x}(m, k)\right]=i Q_{y}(m, k),\left[I_{z}, Q_{x}(m, n)\right]=i(m-n) Q_{y}(m, n),} \\
& {\left[Q_{y}(m, n), I_{z}\right]=i(m-n) Q_{x}(m, n),\left[I_{z}, Q_{z}(m, n)\right]=0 \quad(m \neq n, m \neq k, n \neq k) .}
\end{aligned}
$$

Thus we obtain under the rotating-wave approximation,

$$
\dot{\rho}(t)=G_{1}+G_{2}+G_{3}+G_{4}
$$

with

$$
\begin{aligned}
G_{1}= & -\frac{i}{4} \gamma^{2} H_{1}^{2} \sum_{j} \sum_{k} \alpha_{k} \alpha_{j} \exp (i\{[A(k)+A(j)] t+2 \phi\}) \\
& \times[\rho(t) Q(k, k-1) Q(j, j-1) / A(k)+Q(k, k-1) Q(j, j-1) \rho(t) / A(j) \\
& -Q(k, k-1) \rho(t) Q(j, j-1) / A(k)-Q(k, k-1) \rho(t) Q(j, j-1) / A(j)],
\end{aligned}
$$




$$
\begin{aligned}
G_{2}= & \frac{i}{4} \gamma^{2} H_{1}^{2} \sum_{j} \sum_{k} \alpha_{k} \alpha_{j} \exp (-i\{[A(k)+A(j)] t+2 \phi\}) \\
\times & {[\rho(t) Q(k-1, k) Q(j-1, j) / A(k)+Q(k-1, k) Q(j-1, j) \rho(t) / A(j)} \\
& -Q(k-1, k) \rho(t) Q(j-1, j) / A(k)-Q(k-1, k) \rho(t) Q(j-1, j) / A(j)], \\
G_{3}=- & \frac{i}{4} \gamma^{2} H_{1}^{2} \sum_{j} \sum_{k} \alpha_{k} \alpha_{j} \exp \{i[A(k)-A(j)] t\} \\
& \times[\rho(t) Q(k, k-1) Q(j-1, j)+Q(j-1, j) Q(k, k-1) \rho(t) \\
& \quad-Q(k, k-1) \rho(t) Q(j-1, j)-Q(j-1, j) \rho(t) Q(k, k-1)] / A(k),
\end{aligned}
$$

and

$$
\begin{aligned}
G_{4}= & \frac{i}{4} \gamma^{2} H_{1}^{2} \sum_{k} \sum_{j} \alpha_{k} \alpha_{j} \exp \{i[A(k)-A(j)] t\} \\
& \times[\rho(t) Q(j-1, j) Q(k, k-1)+Q(k, k-1) Q(j-1, j) \rho(t) \\
& \quad-Q(j-1, j) \rho(t) Q(k, k-1)-Q(k, k-1) \rho(t) Q(j-1, j)] / A(j),
\end{aligned}
$$

where $k$ and $j$ are eigenvalues of $I_{z}$ and

$$
\begin{aligned}
A(k) & =\Omega-\Omega_{k-1, k}=\Omega-\left(\left\langle k-1\left|\mathscr{H}_{0}\right| k-1\right\rangle-\left\langle k\left|\mathscr{H}_{0}\right| k\right\rangle\right) \\
& =\Omega-\left[\gamma H_{0}-(2 k-1) \omega_{q}\right]
\end{aligned}
$$

is the frequency offset to the SQ transition $k-1-k$. We consider TQ transition $m-1-m+1$, and assume that the absolute value of frequency offset to the TQ transition

$$
2 \delta=2\left[\Omega-\left(\gamma H_{0}-2 m \omega_{q}\right)\right]
$$

is much smaller than $\gamma H_{1}$. The term $A(k)$ can be rewritten as

$$
A(k)=(2 k-1-2 m) \omega_{q}+\delta
$$

The first and second terms with $k \neq j+1$ in the second set of square brackets in $G_{1}$ in Eq. (A5) vanish owing to the characteristics of $P_{m}$ and $Q(m, n)$. The terms with frequency $|A(k)+A(j)| \gg \gamma H_{1}$ are removed as fast-oscillating ones. Then, we obtain

$$
G_{1}=-\frac{i}{4} \gamma^{2} H_{1}^{2} \exp [2 i(\delta t+\phi)] \alpha_{m} \alpha_{m+1}[\rho(t), Q(m+1, m-1)] / \omega_{q} .
$$

In the derivation we use the approximations $\omega_{q} \pm \delta \sim \omega_{q}$, and $A(k) \pm 2 \delta \sim A(k)$, since $|\delta|$ is much smaller than $\omega_{q}$ and $|A(k)|$.

In a similar way, the term $G_{2}$ is simplified to be

$$
G_{2}=-\frac{i}{4} \gamma^{2} H_{1}^{2} \exp [-2 i(\delta t+\phi)] \alpha_{m} \alpha_{m+1}[\rho(t), Q(m-1, m+1)] / \omega_{q},
$$

and the term $\left(G_{3}+G_{4}\right)$ is reduced to

$$
G_{3}+G_{4}=-\frac{i}{4} \gamma^{2} H_{1}^{2} \sum_{k} \alpha_{k}^{2}\left[\rho(t), Q_{z}(k, k-1)\right] /\left(\Omega-\Omega_{k-1, k}\right) .
$$

Thus, Eq. (A4) becomes 


$$
\dot{\rho}(t)=i \beta[\rho(t), \exp [2 i(\delta t+\phi)] Q(m+1, m-1)+\exp [-2 i(\delta t+\phi)] Q(m-1, m+1)]+i[\rho(t), R],
$$

where

$$
\begin{aligned}
& \beta=\gamma^{2} H_{1}^{2}\left\langle m+1\left|I_{x}^{2}\right| m-1\right\rangle / \omega_{q}, \\
& R=\gamma^{2} H_{1}^{2} \sum_{k} \alpha_{k}^{2} Q_{z}(k, k-1) / 4\left(\Omega-\Omega_{k-1, k}\right) .
\end{aligned}
$$

Using the transformation

$$
\rho^{\dagger}(t)=\exp \left[-i \delta Q_{z}(m+1, m-1) t\right] \rho(t)(\text { H.c. }),
$$

we rewrite Eq. (A13) as

$$
\begin{aligned}
\dot{\rho}^{\dagger}(t)= & i \beta\left[\rho^{\dagger}(t), Q_{x}(m+1, m-1) \cos 2 \phi-Q_{y}(m+1, m-1) \sin 2 \phi\right] \\
& +i \delta\left[\rho^{\dagger}(t), Q_{z}(m+1, m-1)\right]+i\left[\rho^{\dagger}(t), R\right],
\end{aligned}
$$

which is the basic equation quoted in the text.

If the effect of the counter-rotating component of the rf field is taken into account, the term $R$ in Eq. (A16) is replaced by $R+R^{\prime}$, where

$$
R^{\prime}=-\gamma^{2} H_{1}^{2} \sum_{k} \alpha_{k}^{2} Q_{z}(k, k-1) / 4\left(\Omega+\Omega_{k-1, k}\right) .
$$

However, the expectation value of $R^{\prime}$, which gives a Bloch-Siegert shift, is extremely small compared with that of $R$. Then, we neglect the operator $R^{\prime}$ in the following.

The effect of magnetic dipole interaction $\mathscr{H}_{d}$ is considered in an ensemble of spins with Zeeman and interaction Hamiltonians $\mathscr{H}_{0}$ and $\mathscr{H}_{1}(t)$ given by

$$
\begin{aligned}
& \mathscr{H}_{0}=-\gamma H_{0} \sum_{j} I_{j z}+\omega_{q} \sum_{j}\left[I_{j z}^{2}-\frac{1}{3} I(I+1)\right], \\
& \mathscr{H}_{1}(t)=-2 \gamma H_{1} \exp \left(i \mathscr{H}_{0} t\right) \sum_{j} I_{j x} \exp \left(-i \mathscr{H}_{0} t\right) \cos (\Omega t+\phi) .
\end{aligned}
$$

The time development of the ensemble is governed by the following equation:

$$
\dot{\rho}(t)=i\left[\rho(t), \mathscr{H}_{1}(t)+\mathscr{H}_{d}^{\dagger}(t)\right],
$$

where

$$
\mathscr{H}_{d}^{\dagger}(t)=\exp \left(i \mathscr{H}_{0} t\right) \mathscr{H}_{d} \exp \left(-i \mathscr{H}_{0} t\right) .
$$

The effect of the secular part $\mathscr{H}_{d}^{*}$ of the dipole Hamiltonian $\mathscr{H}_{d}^{\dagger}(t)$ can be eliminated by using the transformation $\rho^{*}=U^{-1} \rho U$, where $U=\exp \left(-i \mathscr{H}_{d}^{*} t\right)$. Equation (A19) becomes

$$
\dot{\rho}^{*}(t)=i\left[\rho^{*}(t), \mathscr{H}_{1}^{*}(t)+\mathscr{H}_{d}^{\prime}(t)\right],
$$

where $\mathscr{P}_{1}^{*}(t)=U^{-1} \mathscr{H}_{1}(t) U$ and $\mathscr{H}_{d}^{\prime}(t)=U^{-1}\left[\mathscr{H}_{d}^{\dagger}(t)-\mathscr{H}_{d}^{*}\right] U$. The Hamiltonian $\mathscr{H}_{1}^{*}(t)+\mathscr{H}_{d}^{\prime}(t)$ is a fast oscillating operator and therefore Eq. (A21) can be approximated as

$$
\begin{aligned}
\dot{\rho}^{*}(t)= & -\left[\left[\rho^{*}(t), \int_{0}^{t} \mathscr{H}_{1}^{*}\left(t^{\prime}\right) d t^{\prime}\right], \mathscr{H}_{1}^{*}(t)\right]-\left[\left[\rho^{*}(t), \int_{0}^{t} \mathscr{H}_{1}^{*}\left(t^{\prime}\right) d t^{\prime}\right], \mathscr{H}_{d}^{\prime}(t)\right] \\
& -\left[\left[\rho^{*}(t), \int_{0}^{t} \mathscr{H}_{d}^{\prime}\left(t^{\prime}\right) d t^{\prime}\right], \mathscr{H}_{1}^{*}(t)\right]-\left[\left[\rho^{*}(t), \int_{0}^{t} \mathscr{H}_{d}^{\prime}\left(t^{\prime}\right) d t^{\prime}\right], \mathscr{H}_{d}^{\prime}(t)\right] .
\end{aligned}
$$

The right-hand side of Eq. (A22) can be developed by applying projection operator $\Sigma_{m} P_{m}$. The integrals involved in Eq. (A22) have a common form as

$$
\begin{aligned}
F & =\int_{0}^{t} \exp \left[i A(k) t^{\prime}\right] \exp \left(i \mathscr{C}_{d}^{*} t^{\prime}\right) Q(m, n) \exp \left(-i \mathscr{H}_{d}^{*} t^{\prime}\right) d t^{\prime}, \\
& =\int_{0}^{t} \exp \left[i A(k) t^{\prime}\right] \sum_{n=0}^{\infty}\left(i t^{\prime}\right)^{n}\left[\mathscr{H}_{d}^{*},\left[\mathscr{H}_{d}^{*}, \ldots\left[\mathscr{H}_{d}^{*}, Q(m, n)\right] \ldots\right]\right] / n ! d t^{\prime},
\end{aligned}
$$


where $n$ is the number of $\mathscr{H}_{d}^{*}$ terms. By using integration by parts and neglecting the second- and higherorder terms by assuming that $\left\langle\mathscr{H}_{d}\right\rangle \ll\left\langle\mathscr{H}_{0}\right\rangle$, we obtain

$$
F=-i\left\{\exp [i A(k) t] \exp \left(i \mathscr{P}_{d}^{*} t\right) Q(m, n) \exp \left(-i \mathscr{H}_{d}^{*} t\right)-Q(m, n)\right\} / A(k) .
$$

Using Eq. (A24) and procedures similar to those used to derive Eq. (A13), we derive the following equation from Eq. (A22):

$$
\begin{aligned}
\dot{\rho}(t)=i \beta[ & \rho(t), \sum_{j} Q_{j}(m+1, m-1) \exp [2 i(\delta t+\phi)] \\
& \left.+\sum_{j} Q_{j}(m-1, m+1) \exp [-2 i(\delta t+\phi)]\right]+i[\rho(t), R]+i\left[\rho(t), \mathscr{H}_{d}^{*}\right],
\end{aligned}
$$

where

$$
R=\gamma^{2} H_{1}^{2} \sum_{k} \alpha_{k}^{2} \sum_{j} Q_{j z}(k, k-1) / 4\left(\Omega-\Omega_{k-1, k}\right) .
$$

After the transformation

$$
\rho^{\dagger}(t)=\exp \left[-i \delta \sum_{j} Q_{j z}(m+1, m-1) t\right] \rho(t)(\text { H.c. }),
$$

we finally obtain

$$
\begin{aligned}
\dot{\rho}^{\dagger}(t)= & i \beta\left[\rho^{\dagger}(t), \sum_{j} Q_{j x}(m+1, m-1) \cos (2 \phi)-\sum_{j} Q_{j y}(m+1, m-1) \sin (2 \phi)\right] \\
& +i \delta\left[\rho^{\dagger}(t), \sum_{j} Q_{j z}(m+1, m-1)\right]+i\left[\rho^{\dagger}(t), R\right]+i\left[\rho^{\dagger}(t), \mathscr{H}_{d}^{*}\right]
\end{aligned}
$$

${ }^{1}$ H. Hatanaka, T. Terao, and T. Hashi, J. Phys. Soc. Jpn. $\underline{39}, 835$ (1975).

${ }^{2}$ H. Hatanaka and T. Hashi, Phys. Rev. B $\underline{21}, 2677$ (1980).

${ }^{3}$ E. K. Wolff and M. Mehring, Phys. Lett. 70A, 125 (1979).

${ }^{4}$ For a review, see A. M. Bonch-Bruevich and V. A. Khodovoi, Usp. Fiz. Nauk 93, 71 (1967) [Sov. Phys.-Usp. $10,637(1968)]$.

${ }^{5}$ M. Takatsuji, Phys. Rev. A 4, 808 (1971).

${ }^{6}$ D. Grischkowsky, M. M. T. Loy, and P. F. Liao, Phys. Rev. A 12, 2514 (1975).

${ }^{7}$ For example, H. Hatanaka and T. Hashi, J. Phys. Soc. Jpn. 39, 1139 (1975); A. Pines, S. Vega, D. J. Ruben, T. W. Shattuck, and D. E. Wemmer, Lectures presented at the Ampere Summer School, Pula, Yugoslavia, 1976 (unpublished); M. M. T. Loy, Phys. Rev. Lett. $\underline{36}, 1454$ (1976); H. Hatanaka, T. Ozawa, and T. Hashi, J. Phys. Soc. Jpn. 42, 2069 (1977); D. G. Gold and E. L. Hahn, Phys. Rev. A 16, 324 (1977); H. Hatanaka and T. Hashi, Phys. Lett. 67A, 183 (1978); A. Flusberg, T.
Mossberg, R. Kachru, and S. R. Hartmann, Phys. Rev. Lett. 41, 305 (1978).

${ }^{8}$ R. G. Brewer, and E. L. Hahn, Phys. Rev. A $\underline{11}, 1641$ (1975).

${ }^{9}$ P. F. Liao and J. E. Bjorkholm, Phys. Rev. Lett. 녀, 1 (1975), and references therein.

${ }^{10}$ H. Hatanaka, Ph.D. thesis, Kyoto University, 1977 (unpublished).

${ }^{11}$ S. Vega and A. Pines, J. Chem. Phys. 66, 5624 (1977); A. Wokaun and R. R. Ernst, ibid. 67, 1752 (1977); D. Suwelack, M. Mehring, and A. Pines, Phys. Rev. B 19, 238 (1979).

${ }^{12}$ H. Hatanaka, and T. Hashi, J. Phys. Soc. Jpn. $\underline{50}, 3629$ (1981).

${ }^{13}$ Our papers cited in Ref. 7.

${ }^{14}$ T. Terao and T. Hashi, J. Phys. Soc. Jpn. ㅁ6, 989 (1974).

${ }^{15}$ The statement holds generally for the TQ transitions in $I>1$ systems except for the TQ transitions between levels $m_{z}=-1$ and 1 . 